\title{
DERMATOMIOSITIS Y SÍNDROME DE EVANS ASOCIADO A INFECCIÓN POR HTLV-1
}

\author{
David Loja-Oropeza1,a, Ernesto Zavala-Flores ${ }^{2, b}$, Maricela Vilca-Vasquez $^{3, c}$
}

\begin{abstract}
Paciente mujer de 55 años de edad, natural de Ayacucho, con antecedente de dermatomiositis desde hace 3 años, recibió tratamiento irregular con prednisona. Dos meses antes del ingreso presenta anemia hemolítica autoinmune y púrpura trombocitopénica idiopática, recibió pulsos de metilprednisolona y transfusión de paquetes globulares. Al ingreso, soporosa, mal estado general, marcada pérdida ponderal, deshidratada, livedo reticularis en miembros inferiores, onicodistrofia y onicolisis múltiple en los dedos de ambos pies. Western Blot positivo para HTLV-1. Evoluciona con hipoglicemia recurrente. Reportamos un caso de dermatomiositis y síndrome de Evans presentados en el contexto de una infección por virus linfotrópico humano tipo 1.
\end{abstract}

Palabras clave: Anemia hemolítica autoinmune; Dermatomiositis; Virus 1 linfotropico T humano (fuente: DeCS-BIREME).

\section{DERMATOMIOSITIS AND EVANS SYNDROME ASSOCIATED WITH HTLV- 1 INFECTION}

\begin{abstract}
A 55-year-old female patient, born in Ayacucho, with a history of dermatomyositis for 3 years, who received irregular treatment with prednisone. Two months prior to admission, she presented with autoinmune hemolytic anemia and idiopathic thrombocytopenic purpura. The patient received methylprednisolone pulse therapy and packed red blood cells transfusions. Upon admission, she was drowsy, with a poor overall status, marked weight loss, dehydration, with presence of livedo reticularis in her lower extremities, and onychodystrophy and onycholysis on the toes of both feet. Western blot test was positive for human T-lymphotropic virus type 1 (HTLV-1). The patient evolved with recurrent hypoglycemia. Therefore, we report a case of dermatomyositis and Evans syndrome in the context of an HTLV-1 infection.
\end{abstract}

Key words: Anemia, hemolytic, autoimmune; Dermatomyositis; Human T-lymphotropic virus 1 (source: MeSH NLM).

\section{INTRODUCCIÓN}

La infección por el virus linfotrópico humano (HTLV-1) es una enfermedad emergente en Latinoamérica. Es endémica en países como Brasil, Colombia, Perú y afecta principalmente a poblaciones quechuas, afroamericanos y descendientes de japoneses, alcanzando una prevalencia global de $0,5-5 \%$. En el Perú, la prevalencia varía entre 1,3 a $3,8 \%$, considerándose una infección endémica. La transmisión de HTLV-1 ocurre a través de tres vías: sexual, de madre a niño (sobre todo a través de lactancia materna) y por transfusión de sangre ${ }^{(1,2)}$.

Entre las formas clínicas relacionadas a la infección por HTLV-1 existen tres grupos más o menos bien definidos que incluyen en el primer grupo a enfermedades con manifestaciones inflamatorias como el síndrome de Sjögren, tiroiditis, alveolitis, nefritis intersticial, uveítis, polimiositis-dermatomiositis (PM-DM) y la paraparesia espástica tropical. Un segundo grupo contempla neoplasias como la leucemia de células $T$ del adulto y el linfoma cutáneo. En el tercer grupo se consideran a las infecciones como la sarna noruega, la dermatitis infectiva, la onicomicosis múltiple, la tuberculosis, la hiperinfestación por Strongyloides stercolaris, la lepra y la paracoccidiodomicosis. Estas formas afectan hasta el $5 \%$ de los infectados estando la gran mayoría de los portadores del HTLV-1 asintomáticos (2).

Cuando dos o más enfermedades de base autoinmune, que satisfacen criterios para dichas entidades, se presentan en forma simultánea o una se suma a la otra en la evolución, se denomina síndrome overlap o de solapamiento. Existen ejemplos como la artritis reumatoidea y el lupus eritematoso sistémico (rupus), la esclerosis sistémica y la dermatomiositis (esclerodermatomiositis), la artritis psoriática y el lupus sistémico (psupus), la esclerosis sistémica y el síndrome de Sjögren, la esclerosis sistémica y la artritis reumatoidea, la esclerosis sistémica y la cirrosis biliar

\footnotetext{
Hospital Nacional Arzobispo Loayza. Lima, Perú.

Universidad de San Martín de Porres. Lima, Perú.

Policlínico San Luis. EsSALUD. Lima, Perú.

Médico internista; ${ }^{b}$ estudiante de Medicina Humana; ${ }^{c}$ médico cirujano

Recibido: 09-02-15 Aprobado: 07-07-15
}

Citar como: Loja-Oropeza D, Zavala-Flores E, Vilca-Vasquez M. Sarcoma de Kaposi endemico en un paciente VIH negativo. Rev Peru Med Exp Salud Publica. 2016;33(1):162-67. doi: /10.17843/rpmesp.2016.331.2018 
primaria (síndrome de Reynolds), la polimiositis, el síndrome de Sjögren y la hepatitis autoinmune y varias otras combinaciones ${ }^{(3-5)}$.

También se han descrito el overlap de casos de anemia hemolítica autoinmune asociados a enfermedades del tejido conectivo como lupus eritematoso sistémico, artritis reumatoidea, esclerodermia y más infrecuentemente con dermatomiositis ${ }^{(6,7)}$. Del mismo modo, hay reportes de púrpura trombocitopénica autoinmune relacionados con dermatomiositis ${ }^{(8-10)}$. Pero es extremadamente rara la asociación de síndrome de Evans y dermatomiositis; hechas las revisiones en buscadores y base de datos como PubMed, ScienceDirect, Scopus, Ebsco, Lilacs y Scielo, encontramos solo tres casos que anteceden al nuestro ${ }^{(11,12)}$

El síndrome de Evans es una entidad hematológica autoinmune de rara presentación, caracterizada por la ocurrencia simultánea o secuencial de anemia hemolítica autoinmune y trombocitopenia autoinmune, algunas veces asociada a neutropenia autoinmune. En la mayoría de los casos es de origen idiopático, sin embargo, puede verse asociada a enfermedades linfoproliferativas o sistémicas ${ }^{(10,11)}$.

Se reporta el caso de una paciente con diagnóstico de dermatomiositis que en la evolución desarrolló síndrome de Evans y onicomicosis múltiple asociados a infección por HTLV-1. Discutimos aspectos relacionados a la patogénesis de la infección viral y su probable papel en los fenómenos de autoinmunidad para el desarrollo de enfermedades hematológicas y del tejido conectivo

\section{REPORTE DE CASO}

Paciente mujer de 55 años de edad, natural de Ayacucho, con antecedente de dermatomiositis hace 3 años, en tratamiento irregular con prednisona (20 $\mathrm{mg} / \mathrm{d}$ ). Hipertensión arterial hace 15 años en tratamiento irregular con enalapril.

Cuatro meses antes del ingreso presenta episodio de disminución de fuerza muscular y edema generalizado, disfagia y vómito porráceo por los cuales acude a una Clínica donde le realizan una endoscopía alta y se encuentran úlceras en el fondo gástrico; es dada de alta y se le indica omeprazol.

Tres meses antes del ingreso, al persistir la disminución de la fuerza muscular, decaimiento, hiporexia y pérdida ponderal de aproximadamente $15 \mathrm{~kg}$ de peso, acude a la clínica, donde le realizan nueva batería de exámenes y corroboran el diagnóstico de dermatomiositis. Sale de alta con prednisona $20 \mathrm{mg} /$ día y metotrexate $2,5 \mathrm{mg} / \mathrm{semana}$.
Dos meses antes del ingreso le demuestran anemia hemolítica autoinmune y púrpura trombocitopénica autoinmune, recibe pulsos de metilprednisolona y transfusión de paquetes globulares. Un mes antes del ingreso presenta episodio de hemorragia digestiva alta y baja; en la endoscopía alta se encuentra gastritis erosiva y no evidencia de lesiones en la colonoscopía. Diez días antes del ingreso cursa con hiporexia acentuada, no tolera alimentos por sonda nasogástrica, náuseas, vómitos y estreñimiento. Siete horas antes de acudir por emergencia presenta disartria, sopor y disminución de la fuerza muscular del hemicuerpo izquierdo. Tiene TAC cerebral normal. Se constata hipoglicemia de $16 \mathrm{mg} / \mathrm{dL}$ cuyos síntomas revierten con la infusión de dextrosa.

Al examen físico: PA 80/50 mm Hg; FC 124 por min; FR 22 por min; afebril. Mal estado general, deshidratada. Eritema violáceo facial a predominio palpebral e hipercromía frontal (Figura 1). Llenado capilar menor $2 \mathrm{~s}$, livedo reticularis en miembros inferiores, úlceras de presión interglúteas de $1 \times 2 \mathrm{~cm}$ y de $5 \times 6 \mathrm{~cm}$ grado 3 . Onicodistrofia y onicolisis en los dedos de ambos pies. Pupilas midriáticas fotorreactivas, escleras ictéricas. Torax y pulmones: murmullo vesicular disminuido en bases, crépitos bibasales. Cardiovascular: ruidos cardiacos rítmicos, taquicárdicos de bajo tono e intensidad, no soplos. Abdomen globuloso, blando, depresible, doloroso a la palpación en hemiabdomen derecho, no rebote. Se palpan lesiones elevadas de consistencia dura en pared abdominal. Hígado a $3 \mathrm{~cm}$ debajo recorde costal. Neurológico: Glasgow 10 (03 V2 M5). Asimetría facial izquierda. Fuerza muscular disminuida en hemicuerpo izquierdo. Reflejos osteotendinosos 2/4+. Babinsky bilateral. Clonus presente. No signos meníngeos

\section{EXÁMENES}

Hemoglobina 7,0 gr/dL; glucosa $16 \mathrm{mg} / \mathrm{dL}$; urea $25 \mathrm{mg} /$ dL; gases arteriales: $\mathrm{pH}$ 7,52; PCO2 34,4; PO2 81,1; HCO3 29; electrolitos: $\mathrm{Na} 131 \mathrm{mEq} / \mathrm{L} ; \mathrm{K} \mathrm{2,9} \mathrm{mEq} / \mathrm{L} ; \mathrm{Cl}$ $101 \mathrm{mEq} / \mathrm{L}$; perfil hepático: bilirrubina total 2,6 mg/dL; bilirrubina indirecta 2,0 mg/dL; TGO $159 \mathrm{U} / \mathrm{L} ;$ TGP

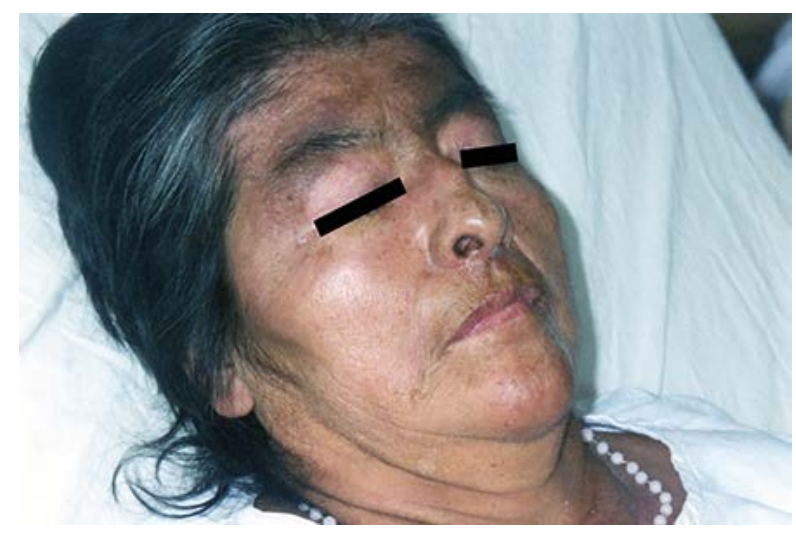

Figura 1. Paciente con eritema facial y signo del heliotropo bilateral 
$179 \mathrm{U} / \mathrm{L}$; Fosfatasa alcalina $227 \mathrm{U} / \mathrm{L}$; calcio 6,6 mg/ dL; CPK 322 U/L; plaquetas $59000 \mathrm{~mm}^{3}$; anticuerpos antiplaquetarios positivos; Coombs directo positivo. HBsAg y anti-HVC negativos. Alfa feto proteína 0,83 ng/mL; CA-125 153,9 Ul/mL (0-35 Ul/mL); CPK 55 U/L; CPK-MB $41 \mathrm{U} / \mathrm{L}$; troponina 0,34 ng/mL; LDH $1816 \mathrm{U} / \mathrm{L}$; beta 2 microglobulina 0,23 mg/dL (<0,22 mg/dL); ELISA HTLV I y II positivo. Western Blot positivo para HTLV-1.

Ecografía abdominal: Iíquido libre en cavidad abdominal. Parénquima hepático hipoecogénico a descartar hepatopatía.

Ecografía transvaginal: útero y ovarios de aspecto involutivo. Líquido libre en cavidad pélvica.

Ecografía de partes blandas: nódulos subcutáneos de aspecto hipoecogénico dolorosos.

Mamografía bilateral: probable proceso fibroquístico de la mama izquierda y múltiples calcificaciones.

Depuración de creatinina: 27,3 mL/min; TGO 384 U/L; TGP 109 U/L.

Anticuerpos antinucleares: positivo. patrón homogéneo $1 / 80$.

Albumina $2,5 \mathrm{~g} / \mathrm{dL}$. Globulina $3,5 \mathrm{~g} / \mathrm{dL}$; creatinkinasa total $5032 \mathrm{U} / \mathrm{L}$ (24-195), VSG $32 \mathrm{mmh}$.

Líquido ascítico negativo a células neoplásicas. Parasitológico en heces seriado negativo.

Baciloscopía en esputo seriado: negativo. Urocultivo: positivo a E. coli. Proteína C reactiva $12,0 \mathrm{ng} / \mathrm{dL}$ (1.10 ng/dL).

Radiografía abdominal: intestino delgado, en yeyuno formaciones de aspecto elevado transparente a manera de defecto de relleno que condiciona falta de adherencia del bario a la mucosa yeyunal. Pliegues dilatados en la región ileal e imágenes de aspecto elevado. Íleon distal con segmentos adelgazados y formaciones de aspecto elevado.

Colonoscopía dentro de límites normales. Hemorroides internas grado $\mathrm{I}$.

Tomografía abdominal: hígado de tamaño y morfología normal, disminuido en su densidad, probablemente por esteatosis. Ligero engrosamiento de las paredes del colon transverso, ángulo esplénico, así como en la región del recto, a descartarse colitis inespecífica.

Tomografía de tórax: discreto incremento del intersticio pulmonar en vidrio esmerilado a nivel de las bases pulmonares de naturaleza inespecífica. Ligero engrosamiento de la pleura costal basal posterior derecho. Cavidades cardiacas con discreta prominencia ventricular izquierda.

Anatomía patológica de biopsia gástrica: gastritis crónica moderada con foco de erosión con actividad aguda moderada. Helicobacter pylori ++/+++.

Anatomía patológica de nódulo subcutáneo: dermatofibroma.

Fue tratada con infusión de dextrosa al 10\%, pulsos de metilprednisolona y luego prednisona a dosis plena, se corrige la hipocalcemia e hipokalemia. Además, recibió ceftriaxona y tratamiento para Helicobacter pylori. La evolución fue estacionaria, y presentó muerte súbita. No se realizó la necropsia por la negativa de los familiares

\section{DISCUSIÓN}

Las enfermedades autoinmunes son un grupo de patologías en las que el organismo pierde la capacidad de diferenciar entre antígenos foráneos y los propios del huésped, lo que condicionaría una autoagresión contra las células del organismo (13,14). Diversos estudios postulan a las infecciones como origen de la autoinmunidad ${ }^{(13)}$, las cuales mediante mecanismos de respuesta cruzada, modificación de las proteínas celulares, del HLA, o generando una respuesta policlonal generarían una alteración de los mecanismos autorreguladores de la respuesta inmune ${ }^{(13)}$.

El papel de algunas infecciones relacionadas a fenómenos de autoinmunidad, se han evidenciado en casos como artritis reumatoide y el parvovirus B-19; lupus eritematoso sistémico (LES) y la enfermedad mixta del tejido conectivo (EMTC) con la infección por Clamydia tracomatis; el virus de la hepatitis $\mathrm{C}$ que se asocia a crioglobulinemia mixta, glomerulonefritis membranoproliferativa. El síndrome de Guillain-Barré, con infección respiratoria o gastrointestinal, así como la espondilitis anquilosante, con la Klebsiella pneumoniae ${ }^{(13,14)}$.

En la patogenia de la infección por HTLV-1, la invasión de los linfocitos CD4+, conduce a grados de inmunosupresión que hará proclive al huésped a enfermedades oportunistas como: tuberculosis, lepra, estrongiloidiasis, escabiosis, entre otras ${ }^{(1,2)}$. En nuestra paciente se evidenció la presencia de onicodistrofia, que es una asociación independiente con infección por HTLV-1. Esta infección podría tener un mayor riesgo para onicomicosis debido al daño de la superficie de la uña y una respuesta inmune mediada por células $T$ disminuido ${ }^{(15)}$. La concentración del virus en plasma es bastante baja. Por lo tanto, la transmisión se da a partir del contacto con linfocitos infectados. El HTLV-1 es un virus que casi nunca sale de las células humanas. A 
diferencia del VIH, la carga viral del HTLV-1 en plasma es indetectable. Para estimar la cantidad de virus se debe procesar la carga proviral. La carga proviral es la proporción de células mononucleares de sangre periférica que contiene el ADN del HTLV-1 en su genoma. Se sabe que las personas con enfermedades asociadas a HTLV-1 suelen tener en promedio valores más altos de carga proviral que los portadores asintomáticos ${ }^{(2)}$.

La infección por el HTLV-1, probablemente induciría una respuesta inmune aberrante. Esta respuesta anómala generaría la producción de autoanticuerpos que reaccionarían contra la membrana del eritrocito produciendo una anemia hemolítica autoinmune ${ }^{(16,17)}$. Del mismo modo, podemos especular que estos autoanticuerpos no se limitaron a la membrana del eritrocito, sino también a la superficie de la membrana plaquetaria, presumiblemente en los receptores IIb-IIla/lb-IIla. La asociación entre estas dos entidades de origen autoinmune es conocida como síndrome de Evans-Fisher, que es una entidad que suele afectar a la población pediátrica, aunque también existen series de casos donde se reporta su aparición en adultos jóvenes y de mediana edad de manera espontánea o secundaria a otras patologías $(11,12,18)$. El síndrome de Evans suele asociarse a otras enfermedades del tejido conectivo, principalmente lupus eritematoso sistémico y esclerodermia, sin embargo, su asociación con dermatomiositis es extremadamente rara ${ }^{(11,12)}$. En nuestro caso la DM precedió a la anemia y trombocitopenia autoinmunes, similar al caso de Chang quien plantea que en pacientes con DM y anemia debería descartarse hemólisis. En el caso de Hay, a la instalación del síndrome de Evans le sucedió el de la DM ${ }^{(11)}$. Jung reporta un caso de DM al que se suma inicialmente la anemia hemolítica y posteriormente la trombocitopenia autoinmunes y reitera que la patogénesis del síndrome de Evans en DM es desconocida, pero que anomalías tanto en la inmunidad humoral como celular se han visto implicadas en el origen del síndrome de Evans. En la patogénesis de la DM cumple un rol más importante la inmunidad humoral que la celular, lo cual se basa en el infiltrado inflamatorio perivascular y perimisial con predominio de linfocitos T CD4 y células $\mathrm{B}^{(12)}$.

Por otro lado, los autoanticuerpos en conjugación con los antígenos, expresados por las proteínas replicadas por el virus, formarían inmunocomplejos que se depositarían en los vasos sanguíneos de pequeño calibre (arteriolas, vénulas y capilares) de los músculos esqueléticos, del tracto gastrointestinal, piel y el tejido celular subcutáneo. Dicho depósito originaría una vasculitis necrotizante que sería la génesis de la DM y de la livedo reticularis presentado por la paciente ${ }^{(19,20)}$.

Algunos reportes señalan la asociación entre DM e infección por HTLV-1, entre ellos, en una corte realizada en Jamaica se encontró que el $85 \%$ (11/13) de los pacientes con PM fueron seropositivos para HTLV-1; en un estudio brasileño se encontró que el 36\% (4/11) de pacientes con mielopatía o paraparesia espástica tropical evidenciaron PM en la biopsia muscular; en la isla de Martinica - Francia, se documentó que un $50 \%(7 / 14)$ de los pacientes con diagnóstico de HTLV1 presentaron DM o PM. Sin embargo, en la serie de Irving de 80 pacientes con enfermedad del tejido conectivo (35 PM-DM) solo cinco tuvieron la prueba de tamizaje para HTLV-1 positiva y un solo caso tuvo la prueba confirmatoria positiva ${ }^{(19,21,22)}$, por lo tanto, es recomendable que los resultados de las pruebas de anticuerpos positivas de tamizaje para HTLV-1 deben ser interpretados con cautela

Otra asociación interesante es el mayor riesgo de neoplasias en DM respecto de la población general, el cual es aun mayor en los mayores de 45-50 años. La tasa de incidencia de DM y cáncer es, en promedio, 20-40\%; y el riesgo relativo respecto de la población general es entre 3 y 7,7 veces mayor. Los factores de riesgo para malignidad en DM incluyen el sexo masculino, la edad mayor a 45-50 años, el desarrollo de lesiones necróticas cutáneas, vasculitis cutánea, el eritema periungueal, cuadro clínico florido, refractariedad al tratamiento, velocidad de eritrosedimentación mayor a 35, proteína C reactiva elevada, marcadores tumorales elevados como CA-125, CA19-9 y albúmina baja, muchos de ellos presentes en nuestra paciente, por lo tanto, podemos postular la presencia de una neoplasia oculta. La DM se asocia principalmente a tumores sólidos más que hematológicos, y dentro de ellos a carcinomas más que sarcomas. Las neoplasias de pulmón, ovario, mama y colon han sido los tumores más frecuentemente reportados. El carcinoma de ovario se presenta mucho más frecuentemente en mujeres con DM que en la población general, alcanzando un riesgo entre 11-17 veces mayor de carcinoma en DM que en la población general. Otros tumores menos frecuentes son el linfoma no Hodgkin, el cáncer de páncreas, de vejiga, de próstata y de riñón. Los marcadores tumorales CA-125 y CA 19-9 son útiles para la detección de tumores sólidos en pacientes con miopatías inflamatorias idiopáticas.

El valor predictivo positivo del CA-125 fue del 62,5\%; mientras que en mujeres sin DM es del 2,3\%. Esto es importante en el diagnóstico de cáncer de ovario, ya que en fases iniciales sigue un curso silente y además son capaces de producir siembras peritoneales aun cuando son de pequeño tamaño e incluso no visibles en los estudios de imágenes (ecografía transvaginal y TAC). Por lo tanto, el hallazgo de CA-125 aumentado con imágenes que demuestren ascitis leve aun sin signos sugestivos de tumor, se debe considerar la exploración de la cavidad abdominal ${ }^{(23,24)}$.

En relación a la hipoglicemia recurrente de nuestra paciente, tuvo como origen probablemente un tumor 
inductor de hipoglucemia. Estos tumores pueden ser de origen mesodérmico (mesoteliomas, fibrosarcomas, leiomiosarcomas) epitelial o hematopoyético (linfoma gastrointestinal, leucemias). La hipoglicemia que producen es de ayuno y se deben al bloqueo de la producción hepática de glucosa debido a la inhibición de la glucogenolisis y gluconeogénesis, bloqueo de la lipolisis en el tejido adiposo, lo que conlleva niveles séricos bajos de ácidos grasos libres y aumento del consumo periférico de glucosa debido tanto a un aumento del consumo de glucosa por el tumor como a un incremento de la captación periférica de glucosa por el músculo esquelético ${ }^{(24)}$.

Respecto de las vías de transmisión del virus del HTLV-1, estas incluyen la lactancia materna prolongada, las relaciones sexuales y las transfusiones sanguíneas. $\mathrm{Si}$ bien es cierto que las transfusiones de sangre total, plaquetas y paquetes globulares contaminados con HTLV1 pueden infectar un $50-70 \%$ de receptores, el virus no es trasmitido a través de subproductos como el plasma fresco congelado o el crioprecipitado. En una serie de 568 mujeres peruanas asintomáticas mayores de 20 años se encontró anticuerpos para el HTLV-1 en el 2,6\% de ellas, de las cuales $13,6 \%$ habían tenido alguna transfusión sanguínea y el 1,2\%, ninguna. El periodo de incubación es variable, pero el inicio de las manifestaciones clínicas puede llevar 20 a 30 años como en la paraparesia espástica tropical. En Latinoamérica, las tasas de seropositividad para HTLV-1 entre donantes de sangre alcanza 0,1 a 2\%. En el Perú, desde Junio de 1998 el Ministerio de Salud ha dispuesto el despistaje de HTLV1 obligatorio en los bancos de sangre entre los donantes de sangre con lo cual se evitan aproximadamente 4000 transfusiones infectadas por año ${ }^{(2,25,26)}$ Por lo tanto, es poco probable que en nuestra paciente, la transmisión del virus se haya producido por esta vía, y más bien creemos que la procedencia de la paciente de una zona de alta endemicidad para el HTLV-1 como es Ayacucho con patrones culturales que alientan la lactancia materna prolongada hayan sido determinantes para la infección viral.

El tratamiento de la DM-PM incluye el uso de corticoides y citotóxicos (azatioprina, metotrexato, ciclofosfamida); sin embargo, algunospacientesnorespondensatisfactoriamente a la terapia convencional y se requieren otras terapias como inmunoglobulinas endovenosas, tacrolimus, ciclosporina o rituximab, en esquemas combinados $(27,28)$.

Izumi en Japón mostró éxito en el control de la miopatía inflamatoria con tacrolimus ( $3 \mathrm{mg} /$ día) y el posterior uso de ciclosporina A (150 mg/día) junto con prednisolona (20 mg/día) en una paciente con DM y síndrome de Sjögren asociado a infección por HTLV-1 ${ }^{(28)}$; del mismo modo, Jung en Corea obtuvo una buena evolución posterior al uso de ciclosporina oral ( $3 \mathrm{mg} / \mathrm{kg} / \mathrm{día}$ ) junto con prednisona ( $1 \mathrm{mg} / \mathrm{kg} /$ día) en el manejo de edema subcutáneo generalizado en una paciente de 52 años con DM y síndrome de Evans ${ }^{(12)}$. Otro caso reportado por Chang en Corea evidencia buenos resultados en el manejo de estas dos entidades con el uso de inmunoglobulinas intravenosas $(400 \mathrm{mg} / \mathrm{kg} / \mathrm{día}$ por 5 días consecutivos) asociado a ciclofosfamida $(2,2 \mathrm{mg} /$ $\mathrm{kg}$ ) y prednisolona oral (70 mg/día) ${ }^{(29)}$.

En conclusión, la asociación entre DM y síndrome de Evans en el contexto de una infección por HTLV-1 es inusual, por lo que se debería descartar la existencia fenómenos autoinmunes comunes que justifiquen la simultaneidad de estas comorbilidades hematológicas y del tejido conectivo, probablemente desencadenadas por este agente viral, cuya sospecha debe basarse sólidamente en el entorno epidemiológico de alta endemicidad con factores como sexo de riesgo, transfusiones sanguíneas y lactancia materna prolongada. Asimismo, el diagnóstico de infección por HTLV-1 debe incluir las pruebas de tamizaje seguidas de las pruebas confirmatorias. La débil asociación de los reportes de casos de infección por HTLV-1 con PM-DM en el futuro deben ser relevados por estudios con series grandes que establezcan una clara vinculación.

Contribuciones de autoría: DLO, MVV y EZF participaron en la concepción del artículo, MVV contribuyó con el reporte y presentación inicial del caso, DLO Y EZF participaron en la redacción del artículo, DLO Y EZF participaron en la revisión crítica del artículo, todos los autores aprobaron el artículo para publicar.

Fuentes de financiamiento: autofinanciado.

Conflictos de interés: los autores declaran no tener conflictos de interés.

\section{REFERENCIAS BIBLIOGRÁFICAS}

1. Biglione M, Berini C. Aportes $y$ consideraciones sobre la infección por los Virus Linfotrópicos T Humanos tipo $1 \mathrm{y}$ 2 en Argentina. Rev Argent Salud Pública. 2013;4(14):32-7.

2. Gotuzzo E, González E, Verdonck K, Mayer E, Ita F, Clark D. Veinte años de investigación sobre HTLV-1 y sus complicaciones médicas en el Perú: perspectivas generales. Acta méd peruana. 2010;27(3):196-203.

3. Balbir-Gurman A, Braun-Moscovici Y. Scleroderma overlap síndrome. Isr Med Assoc J. 2011;3(1): 14-20

4. Parente J, Mathurdas P, Wandscheneider L, Aranha J, Siopa L. Síndroma de overlap.
Acta Med Port. 2011; 24(S3): 719-24.

5. Jury EC, D’Cruz D, Morrow WJ Autoantibodies and overlap syndromes in autoimmune rheumatic disease. J Clin Pathol 2001;54(5):340-7. doi:10.1136/ jcp.54.5.340

6. Hardman CM, Garioch JJ, Leonard JN, Ozanne 
C, Abdalla SH. Autoimmune haemolytic anaemia associated with dermatomyositis. Clin Exp Dermatol.1996; 21(6):437-9. doi: 10.1111/j.1365-2230.1996.tb00150.x

7. Andrews J, Hall MA. Dermatomyositisscleroderma overlap syndrome presenting as autoimmnue haemolytic anaemia. Rheumatology 2002;41(8):956-8. doi: $10.1093 /$ rheumatology/41.8.956

8. Cooper C, Fairris G, Cotton DW, Steart P, Barth JH. Dermatomyositis associated with idiopathic thrombocytopenia. Dermatologica. 1986;172(3):173-6.

9. Okamoto H, Soejima M, Takeuchi M, Tateishi M, Terai C, Hara M, et al. Dermatomyositis associated with autoimmune idiopathic thrombocytopenia and anti-Ku antibody. Mod Rheumatol. 2004;14(2):187-90. doi: 10.1007/s10165004-0290-9

10. Nozomu K, Kiminori Y, Yasuyo Y, Shuhei N, Hiroshi W, Rumi F, et al. Anti-Ku antibodypositive scleroderma-dermatomyositis overlap syndrome developing Graves' disease and immune thrombocytopenic purpura. Intern Med 2002;41(12):1199-203. doi: 10.2169/internalmedicine.41.1199

11. Hay EM, Makris M, Winfield J, Winfield DA. Evans' syndrome associated with dermatomyositis. Ann Rheum Dis. 1990;49(10):793-4.

12. Jung KD, Kim PS, Park HY, Kim CR, Byun JY, Lee DY, et al. Dermatomyositis associated with generalized subcutaneous edema and Evans syndrome. J Am Acad Dermatol. 2012;66(1):144-7. doi: 10.1016/j.jaad.2009.10.035

13. Blasco Patiño F. La infección como origen y desencadenante de enfermedades autoinmunes. An. Med. Interna (Madrid). 2002;19(1):44-8.
14. Torres S, Martínez Z. Factores genéticos, inmunológicos y ambientales asociados a la autoinmunidad. Rev Cubana Invest Bioméd. 2011;30(4):501-10.

15. Mayer EF, Ita F, Gonzalez E, Verdonck K, Bravo F, Clark D, Gotuzzo E. Association between onychodystrophy and human T-lymphotropic virus type 1 infection. Int J Infect Dis. 2013;17(5):e312-6. doi: 10.1016/j.ijid.2012.11.009

16. Zarandona JM, Yazer MH. The role of the Coombs test in evaluating hemolysis in adults. CMAJ. 2006;174(3):305-7. doi: 10.1503/cmaj.051489

17. Alfonso A, Bencomo A. Tratamiento de las anemias hemolíticas autoinmunes. Rev Cubana Hematol Inmunol Hemoter. 2013;29(4):327-39.

18. Dosi RV, Ambaliya AP, Patell RD, Patil RS, Shah PJ. A case report of Evans Syndrome. Indian $\mathrm{J}$ Med Sci. 2012;66(3-4): 82-5. doi: 10.4103/00195359.110920

19. Bohan A, Peter JB. Polymiositis and dermatomyositis (first of two parts). N Eng J Med. 1975;292(7):344-7. doi: 10.1056/ NEJM197502132920706

20. Maldonado C, Martínez, V, Navarrete G, Ríos HY, Martínez J. Dermatomiositis idiopática primaria. Dermatología Rev Mex. 2012;56(5):308-17.

21. Martin F, Taylor GP, Jacobson S. Inflammatory manifestations of HTLV-1 and their therapeutic options. Expert Rev Clin Immunol. 2014;10(11):1531-46. doi: 10.1586/1744666X.2014.966690

22. Irvine W, White $P$, Cambridge G. Antibodies to HTLV-I in sera from patients with connective tissue diseases. Annals Rheum Dis, 1989;48(1):80.
23. Candiz M, D’Atri G. Dermatomiositis asociada a neoplasias. Dermatol Argent. 2011;17(6):434-40.

24. Forga L, Anda E, Martínez de Esteban JP. Síndromes hormonales paraneoplásicos. Anales Sis San Navarra. 2005;28(2):213-26.

25. Gotuzzo E. Risk of transfusion-transmitted human T-cell lymphotropic virus-type I in Latin America. Int J Infec Dis. 2000;4(2):5961. doi: 10.1016/S1201-9712(00)90094-8

26. Santos N, Bengoa E, De los Santos-Fortuna E, Caterino-de-Araujo A. Confirming the presence of HTLV-1 infection and the absence of HTLV-2 in Blood donors from Arequipa, Peru. Rev Inst Med trop S Paulo. 2009;51(1):25-9. doi: 10.1590/S003646652009000100005

27. Perosa F, Prete M, Racanelli V, Dammacco F. CD20-depleting therapy in autoimmune diseases: from basic research to the clinic. J Intern Med. 2010;267(3):260-77. doi: 10.1111/j.1365-2796.2009.02207.x

28. Izumi Y, Kojima H, Koga Y, Yokota $\mathrm{K}$, Mori $\mathrm{H}$, Ohno $\mathrm{T}$, et al. Successful treatment of HTLV-1related overlap syndrome using tacrolimus. Intern Med. 2011;50(17):1849-53. doi: 10.2169/internalmedicine.50.5013

29. Chang DK, Yoo DH, Kim TH, Jun JB, Lee $\mathrm{IH}$, Bae SC, et al. Induction of remission with intravenous immunoglobulin and cyclophosphamide in steroid-resistant Evans' syndrome associated with dermatomyositis. Clin Rheumatol. 2001;20(1):63-6.

Correspondencia: David Loja Oropeza

Dirección: Reynolds 114-101 Lima 41

Correoelectrónico:davidloja@hotmail.com 